\title{
Should We Pay Attention to the Fibular Segmental Fracture in the Management of Leg Fractures?
}

\author{
Nima Bagheri,, Seyyed Saeed Khabiri, ${ }^{2,}{ }^{,}$SM Javad Mortazavi, ${ }^{1}$ and Mahmoud Farzan ${ }^{1}$ \\ ${ }^{1}$ Orthopedic Surgeon, Joint Reconstruction Research Center, Tehran University of Medical Sciences, Tehran, Iran \\ ${ }^{2}$ Resident of Orthopedic Surgery, Joint Reconstruction Research Center, Tehran University of Medical Sciences, Tehran, Iran \\ "Corresponding author: Seyyed Saeed Khabiri, Orthopedic Resident, Department of Orthopedic Surgery, Imam-Khomeini Hospital, Tehran University of Medical Sciences, \\ Keshavarz Blvd, P.O. Box 14197-33141, Tehran. Tel: +98-2166581586, E-mail: saeed.khabiri@gmail.com
}

Received 2016 August 01; Revised 2016 August 30; Accepted 2016 September 19.

\begin{abstract}
Background: Fibular fractures are the most common associated fractures with tibia injuries. The current study aimed at collecting data on segmental fibular fractures in the patients and investigating the prognostic and functional significance of this injury pattern in outcomes.

Methods: The charts were retrospectively reviewed from 2013 to 2016 to find patients with segmental fibular fractures. Patients' history, physical examinations, initial radiographic findings, surgical reports, and follow-up data were evaluated. The patients were also assessed for union status, infection, limb shortening, and complications. Fractures were defined as segmental when the bone was disrupted in 2 points and a segment of bone with circumferential cortices was apart from the main bone proximally and distally. Results: During the 3-year period of the current study, out of 212 patients with leg fracture (tibiofibular fracture) treated in Imam Khomeini hospital, 17 patients (8\%) had segmental fibular fractures. In the observed fibular fractures, the number of fracture fragments was between 3 and 6 segments. After 6 months, the rate of union was $70 \%$ in the tibia and $88 \%$ in the fibula. Overall, 6 patients needed re-operation, 1 of them had below knee amputation, and 5 other had surgeries because of tibial nonunion.

Conclusions: The current study results showed that presence of a segmental fibular fracture may be associated with higher rates of complications. Fixation of the fibula in this situation can lead to nonunion. However, more studies are needed to approve or reject this conclusion.
\end{abstract}

Keywords: Segmental Fracture, Fibula Fracture, Outcomes

\section{Background}

Fractures of the tibia and fibula are relatively common and recognized as serious and debilitating injuries for centuries (1). Fibular fractures are the most common associated fractures with tibia injuries. Despite the high prevalence of fibular fractures, it receives less attention by orthopedic surgeons. About 7\% to 15\% of body weight is tolerated by fibula and it plays an important role in knee and ankle stability (2). Although isolated fibular fractures occur in only $0.6 \%$ of leg fractures (1), tibia injuries are frequently accompanied by fibular fractures, especially in the context of high-energy trauma. In a literature review, most of the articles found about the fibular fractures are the ones associated with ankle injuries and in the zone of the lateral malleolus. It seems that except lateral malleolus fractures, most other fibular injuries do not alter the therapeutic approach, although it might have some prognostic values. According to the current study findings, some tibiofibular fractures had a segmental fragment. According to the data presented by Ramasamy, about $16 \%$ of fibular fractures are segmental (3). There is a paucity of the literature in the field of fibular fractures. Paying attention to these frac- tures in addition to tibia fractures, and reporting the data can fill this defect. The current study aimed at collecting data on fibular segmental fractures in the patients and investigating the prognostic and functional significance of this injury pattern in outcomes.

\section{Methods}

The current study retrospectively reviewed the charts of all tibia and/or fibula fractures admitted to Imam Khomeini hospital, Tehran, Iran, from 2013 to 2016, to find patients with segmental fibular fractures. All tibiofibular fractures were include and all leg X-rays reviewed; after selecting the patients with segmental fibular fractures, patients' history, physical examinations, initial radiographic findings, surgical reports, and follow-up data were assessed. The patients were also assessed for union status, infection, limb shortening, and complications. During the follow-up, anteroposterior and lateral full-length radiographs of the injured legs at the last visit were obtained. Fractures were defined as segmental when the bone was disrupted in 2 points and a segment of bone with circum- 
ferential cortices was apart from the main bone proximally and distally (4).

\section{Results}

During the 3-year period of the study, out of 212 patients with leg fracture (tibiofibular fracture) that were treated in Imam Khomeini Hospital, 17 (8\%) cases had segmental fibular fractures. Fourteen patients were male. Mean age was $35.6 \pm 3.9$ years, ranging from 23 to 61 . The mechanism of injury for all the patients was road accidents (11 motor vehicle accidents, 6 pedestrians hit by a motor vehicle). Eleven patients had open tibia fractures; none had open fibular fractures. Two patients needed a skin graft and no patient needed vascular surgery. Mean follow-up was $22 \pm 7.58$, ranged 12 to 36 months. Six patients had injuries associated with other limbs or organs including intracranial hemorrhage needing neurosurgical intervention $(\mathrm{n}=1)$, contralateral proximal humerus fracture $(\mathrm{n}=$ 1), ipsilateral olecranon fracture $(n=1)$, ipsilateral femoral fracture $(n=1)$, and ipsilateral metatarsal fractures $(n=2)$.

Open reduction internal fixation(ORIF) was performed in 2 of the fibular fractures (only the distal fracture line of the segmental fracture), as the fracture was in the lateral malleolus zone. The other 15 fractures were not fixed.

Tibia fractures in the patients were repaired by different methods according to fracture type and soft tissue status: 7 by intramedullary nailing, 5 by plate fixation, and 5 by external fixations. In the external fixation group, 3 patients underwent secondary nailing and 2 had external fixator as definitive treatment (Figures 1 to 3 ). In the fibular fractures, the number of fracture fragments was between 3 and 6 segments with no correlation with the number of tibia fractures fragments (based on Pearson correlation analysis) (Table 1).

The anatomic locations of tibia fractures were in proximal third $(\mathrm{n}=3)$, middle third $(\mathrm{n}=7)$, and distal third $(n=7)$. One of the patients had hemophilia and compartment syndrome, and eventually underwent a below knee amputation because of severe necrosis and resistant infection. After 6 months from injury, 5 patients (29.4\%) had tibia nonunion (that was infected in 4 cases) that underwent surgery/surgeries for infection treatment and nonunion repair. Of these 5 patients, 2 (11.7\%) also had fibular nonunion; both had previous ORIF of fibular fracture (fibular fracture fixed only in these patients internally).

Out of the 17 patients, 7 (41\%) needed another surgery: 5 due to nonunion (all with infected nonunion, except one), 1 for osteomyelitis (infected union), and another one for the unviable limb. Tibial nonunion repaired in all patients, 12 months after the injury. All the patients were ambulatory at the end of follow-up. The knee range of motion
(ROM) was normal in all patients, but ankle ROM was restricted severely in 1 patient.

\section{Discussion}

In the review of the literature, only 1 paper discussed segmental fibular fractures in association with a tibia fracture. The current study used PubMed database for the literature review and used "Fractures, Bone" and "Fibula" and segmental as keywords. Ramasamy suggested that segmental fibular fractures are associated with high energy trauma, more open wounds, and more complications (nonunion, infection, and skin loss) compared with tibia fracture with a simple fibula fracture (3). There was no finding on the management and employment of approaches for such patients in the mentioned article.

In Ramasamy's article, open fracture in the group with non-segmental fibula fractures was $53 \%$. In the current study, the prevalence of open fracture was $64.7 \%$ in 17 patients with a fibular segmental fracture. It was comparable to the results reported by Ramasamy. The current study, the rate of open injuries in fibular fractures was significantly higher than that of tibia fractures with no segmental fracture $(P$-value $=0.031)$. It may implicate that these fractures are a sign of high-energy trauma.

The incidence of infection in the current study patients was also similar to that of Ramasamy (35\% vs. $34 \%$, respectively). After 6 months, the rate of union was 70\% in the repaired tibia and $88 \%$ in the repaired fibula. In the previous literature, the rate of tibial union in surgical treatment and the closed fracture was $92 \%$ to $99 \%$ (5-7). The rate of union in open fractures was $76 \%$ to $94.7 \%(5,8,9)$. In comparison, in the current study nonunion rate was higher than the ones with closed fracture ( $\mathrm{P}$-value $=0.039)$, but not significantly superior to open fracture $(\mathrm{P}$-value $=0.320)$.

There is no evidence based on the proper treatment of fibular fractures (including simple or segmental) in patients with tibiofibular fractures. In 1982, Bostman suggested a displacement more than one-third of diaphyseal diameter as an indication for fibula fixation (10).

Some authors concluded that fibular fixation, out of the range of syndesmosis and lateral malleolus, has no clinical and simulation value and there is no need for fibula osteosynthesis (11-13).

In 1998, Williams et al. did not recommend fibula fixation in patients with tibia plateau fractures as they did not find a clinical significance and it was also associated with more complications (14). Whorton et al. said that fixation of the fibula in patients with tibia-fibula open fracture had no effect on union and alignment, but fibula fracture in the zone of syndesmosis and lateral malleolus were not included in this study (15). Barei et al., reported that 

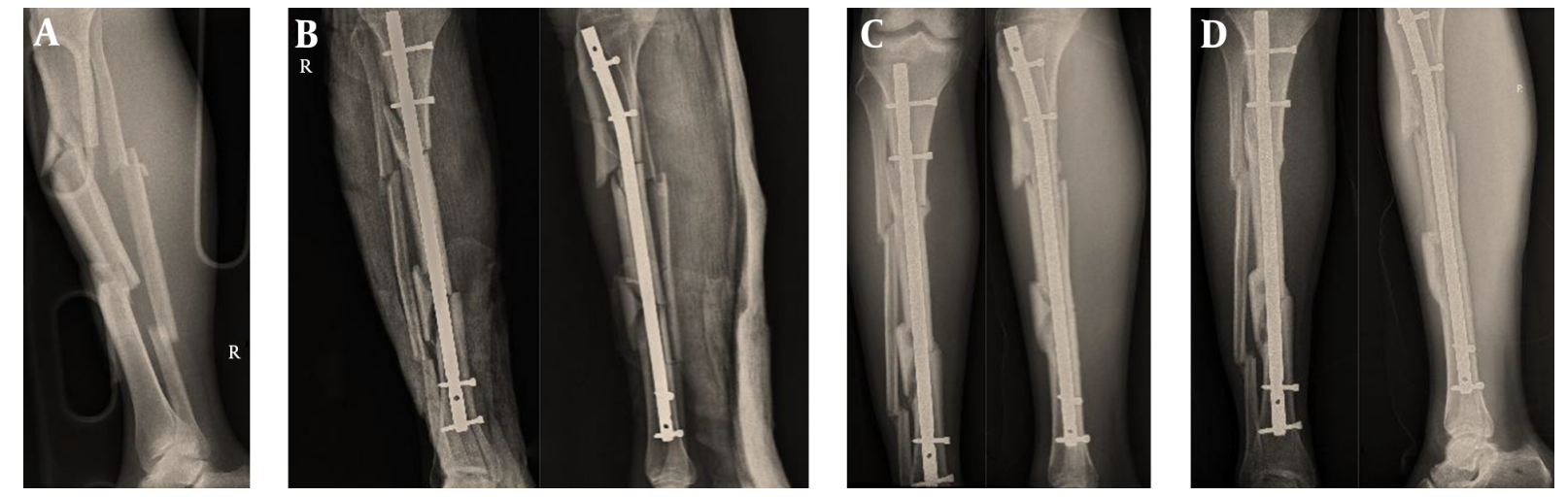

Figure 1. Leg Radiography: A) Preoperation, B) Postoperation, C) Two Months, and D) Six Months After Surgery
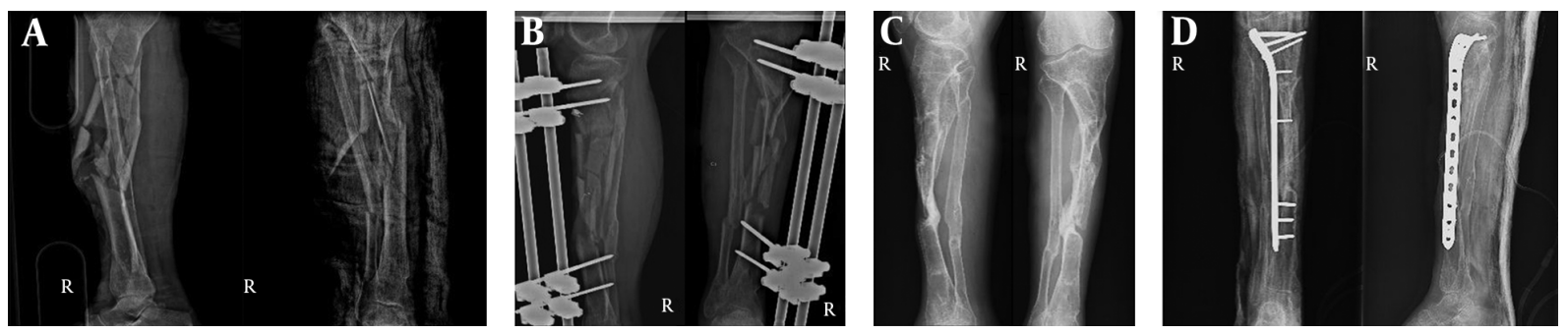

Figure 2. Leg Radiography: A) Preoperation, B) One Month, C) Six Months, and D) Nine Months After Surgery
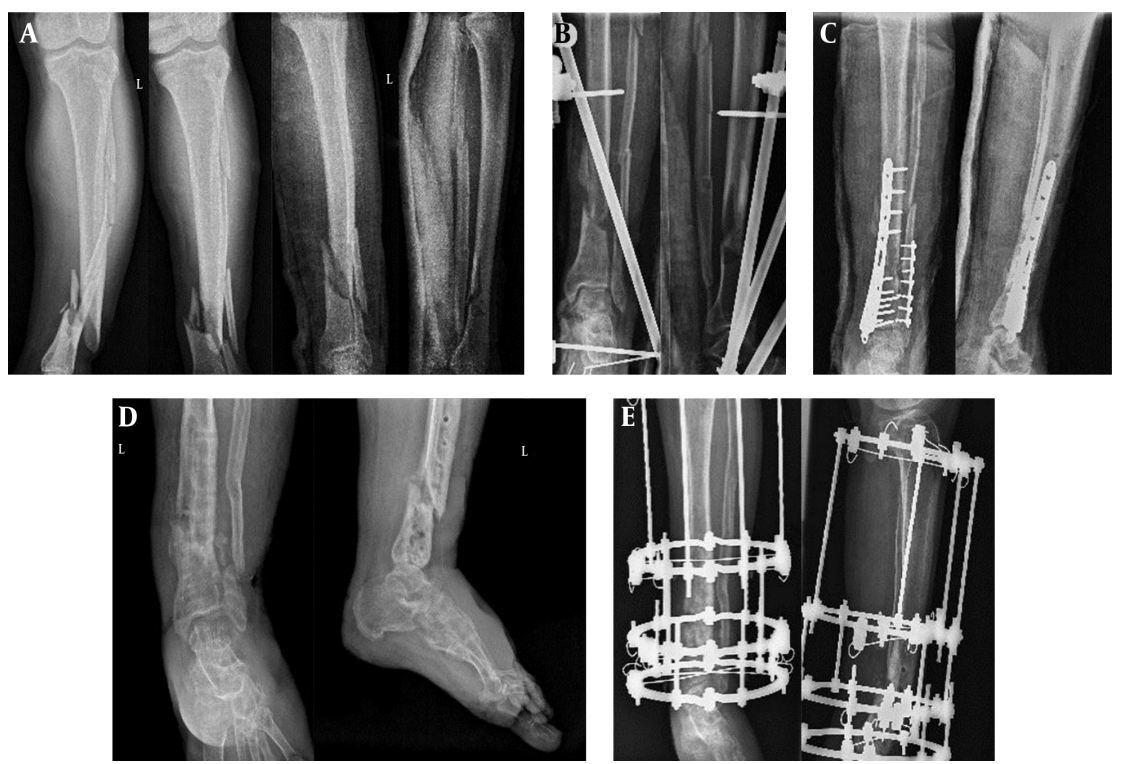

Figure 3. A) Preoperation, B) Primary Temporary Fixation, C) Permanent Fixation, D) After 6 Months, E) Illizarov Fixation

from 72 patients who underwent IM nailing for tibia fracture, 25 had concurrent fibular fixation by the plate, and the others had no fixation. They concluded that after 12 weeks, the group with internal fixation of fibula had bet- 


\begin{tabular}{|c|c|c|c|c|c|c|}
\hline number & Age, years & Gender & Fx Type & Tibia Fixation & Tibia Fragment & Fibula Fragment \\
\hline $\mathbf{1}$ & 37.00 & Male & Open fx & External fixation & 2.00 & 3.00 \\
\hline 2 & 38.00 & Female & Close $\mathrm{fx}$ & Intramedullary fixation & 2.00 & 3.00 \\
\hline 3 & 24.00 & Male & Open fx & Intramedullary fixation & 2.00 & 3.00 \\
\hline 4 & 26.00 & Male & Close $\mathrm{fx}$ & Intramedullary fixation & 4.00 & 3.00 \\
\hline 5 & 37.00 & Female & Open $\mathrm{fx}$ & Intramedullary fixation & 2.00 & 3.00 \\
\hline 6 & 61.00 & Male & Close $\mathrm{fx}$ & Intramedullary fixation & 2.00 & 3.00 \\
\hline 7 & 52.00 & Male & Close $\mathrm{fx}$ & Plate & 2.00 & 3.00 \\
\hline 8 & 39.00 & Male & Open fx & Intramedullary fixation & 2.00 & 3.00 \\
\hline 9 & 54.00 & Male & Open fx & Plate & 2.00 & 5.00 \\
\hline 10 & 31.00 & Male & Close $\mathrm{fx}$ & Intramedullary fixation & 2.00 & 3.00 \\
\hline 11 & 36.00 & Male & Open fx & Plate & 3.00 & 3.00 \\
\hline 12 & 24.00 & Male & Open fx & External fixation & 3.00 & 3.00 \\
\hline 13 & 56.00 & Male & Open fx & External fixation & 2.00 & 4.00 \\
\hline 14 & 26.00 & Male & Open $\mathrm{fx}$ & External fixation & 2.00 & 3.00 \\
\hline 15 & 23.00 & Male & Open $\mathrm{fx}$ & Plate & 2.00 & 3.00 \\
\hline 16 & 32.00 & Female & Close $\mathrm{fx}$ & Plate & 2.00 & 3.00 \\
\hline 17 & 27.00 & Male & Open fx & External fixation & 4.00 & 3.00 \\
\hline
\end{tabular}

ter alignments, but the authors noted that the majority of fixation group had fractures in the lateral malleolus and syndesmotic regions (16).

In the current review, in 2 cases (\#9 and \#12) according to the above literature indications, only the fracture line in the distal part of fibula by plate were fixed. Despite the expectations, nonunion happened in fixation site and maybe if the fixation was performed by intramedullary device or bone graft was used, these nonunion results could be avoided. In these 2 patients, fibula was segmented into 3 and 5 separate parts. Both of their fractures were open and fixed by one-third tubular plates, after primary temporary external fixator. Nonunion of tibia also occurred in both patients. It is interesting that all of the fractures that happened proximal to fixation site in the fibula were united perfectly. Therefore, infection was ruled out and plate removal was done; then, illizarov fixation was performed with bone graft for both patients. After 4 months all particles united, eventually. The current study had its limitations, the number of cases was few and the type of study was designed retrospectively. More studies are required to demonstrate fixation usefulness in segmental fibular fractures.

\subsection{Conclusion}

The current study results showed that presence of a segmental fibular fracture may be associated with higher rates of complications. Fixation of the fibula in this situation can lead to nonunion. However, more studies are needed to approve or reject this conclusion.

\section{Acknowledgments}

Authors would like to acknowledge their gratitude to Dr. Sorena Keihani for critical English editing of the manuscript and his assistance in statistical analysis.

\section{Footnotes}

Conflicts of interests: The authors declared no conflict of interest exists.

Funding/Support: The current study was financially supported by Joint Reconstruction Research Center.

\section{References}

1. Tornetta P, Court-Brown CM, Heckman JD, McQueen MM, Ricci WM, Flynn JM. Rockwood and green's fractures in adults + rockwood and wilkin's fractures in children. Lippincott Williams and Wilkins; 2014. 
2. Goh JC, Mech AM, Lee EH, Ang EJ, Bayon P, Pho RW. Biomechanical study on the load-bearing characteristics of the fibula and the effects of fibular resection. Clin Orthop Relat Res. 1992(279):223-8. doi: 10.1097/00003086-199206000-00028. [PubMed:1600659].

3. Ramasamy PR. The significance of segmental fibular fractures in the management of associated tibial fractures. Injury. 2009;40(3):327-32. doi:10.1016/j.injury.2008.07.014. [PubMed: 19178909].

4. Rockwood and Green's fractures in adults. Philadelphia: Lippincott Williams \& Wilkins; 2006.

5. Jensen JS, Hansen FW, Johansen J. Tibial shaft fractures. A comparison of conservative treatment and internal fixation with conventional plates or AO compression plates. Acta Orthop Scand. 1977;48(2):204-12. doi: 10.3109/17453677708985136. [PubMed: 868506].

6. Puno RM, Teynor JT, Nagano J, Gustilo RB. Critical analysis of results of treatment of 201 tibial shaft fractures. Clin Orthop Relat Res. 1986(212):113-21. doi: 10.1097/00003086-198611000-00013. [PubMed: 3769277].

7. Im GI, Tae SK. Distal metaphyseal fractures of tibia: a prospective randomized trial of closed reduction and intramedullary nail versus open reduction and plate and screws fixation. J Trauma. 2005;59(5):1219-23. doi: 10.1097/01.ta.0000188936.79798.4e. [PubMed: 16385303] discussion 1223.

8. Kakar S, Tornetta P3. Segmental tibia fractures: a prospective evaluation. Clin Orthop Relat Res. 2007;460:196-201. doi: 10.1097/BLO.0b013e318050a3fo. [PubMed:17353796].

9. Ruedi T, Webb JK, Allgower M. Experience with the dynamic compression plate (DCP) in 418 recent fractures of the tibial shaft. Injury. 1976;7(4):252-7. doi: 10.1016/S0020-1383(75)80002-7. [PubMed: 1278983].
10. Bostman O, Hanninen A. The fibular reciprocal fracture in tibial shaft fractures caused by indirect violence. Arch Orthop Trauma Surg. 1982;100(2):115-21. doi: 10.1007/BF00462348. [PubMed: 7138248].

11. Kumar A, Charlebois SJ, Cain EL, Smith RA, Daniels AU, Crates JM. Effect of fibular plate fixation on rotational stability of simulated distal tibial fractures treated with intramedullary nailing.J Bone Joint Surg Am. 2003;85-A(4):604-8. doi: 10.2106/00004623-200304000-00003. [PubMed: 12672833].

12. Morin PM, Reindl R, Harvey EJ, Beckman L, Steffen T. Fibular fixation as an adjuvant to tibial intramedullary nailing in the treatment of combined distal third tibia and fibula fractures: a biomechanical investigation. Can J Surg. 2008;51(1):45-50. [PubMed:18248705].

13. Vasanad GH, Antin SM, Akkimaradi RC, Policepatil P, Naikawadi G. The Role of Fibular Fixation in Distal Tibial Fractures. J Clin Diagn Res. 2016;10(4):RC12-4. doi: 10.7860/JCDR/2016/7249.7833. [PubMed: 27190908].

14. Williams TM, Marsh JL, Nepola JV, DeCoster TA, Hurwitz SR, Bonar SB. External fixation of tibial plafond fractures: is routine plating of the fibula necessary?. J Orthop Trauma. 1998;12(1):16-20. doi: 10.1097/00005131-199801000-00003. [PubMed: 9447514].

15. Whorton AM, Henley MB. The role of fixation of the fibula in open fractures of the tibial shaft with fractures of the ipsilateral fibula: indications and outcomes. Orthopedics. 1998;21(10):1101-5. [PubMed: 9801234].

16. Barei DP, Nork SE, Bellabarba C, Sangeorzan BJ. Is the absence of an ipsilateral fibular fracture predictive of increased radiographic tibial pilon fracture severity?. J Orthop Trauma. 2006;20(1):6-10. doi: 10.1097/01.bot.0000189589.94524.ff. [PubMed: 16424803]. 